\title{
A Flexible Policy Using Failure Rate to Forecast the Repairable Spare Part Stock out Time
}

\author{
Hui Ming Wee \\ Department of Industrial and Systems Engineering, \\ Chung Yuan Christian University, \\ Tao-Yuan, 32023, Taiwan, \\ e-mail:weehm@cycu.edu.tw
}

\author{
Kuo Hsing Wu \\ Department of Industrial and Systems Engineering, \\ Chung Yuan Christian University, \\ Tao-Yuan, 32023, Taiwan, \\ e-mail: paul.kh.wu@gmail.com
}

\author{
Yosef Daryanto \\ Department of Industrial and Systems Engineering, \\ Chung Yuan Christian University, \\ Tao-Yuan, 32023, Taiwan, \\ Department of Industrial Engineering, \\ Universitas Atma Jaya Yogyakarta, \\ Yogyakarta, 55281, Indonesia, \\ e-mail: daryanto@mail.uajy.ac.id
}

\begin{abstract}
Since spare part availability impacts the fixing rate for defective device, maintaining an acceptable spare part availability level is critical for improving customer satisfaction level. Spare part demand is typically unpredictable due to varying service requests. Most companies set a fix period for maintenance and repair. This fixed period of repair model is a waste if the device is in a good condition. To improve the system, this study proposes a flexible return policy using failure rate to forecast the possible stock out time point. Once the specific point is found, the least repair turnaround time (TAT) is set for defective spare part shipment. By this mean, the repaired spare part will arrive at the warehouse to replenish the stock. Similar scenario also applies to the out of warranty period for the dropping service demand. For practical industrial application, this model also considers the last time buy and the defective spare part scrap rate practices.
\end{abstract}

Keywords-inventory management; spare part; forecasting; part availability

\section{INTRODUCTION}

The reason why spare part management is so importance in promoting and maintaining customer satisfaction is because it supports product after sales service. After sales service performance, specifically the repairing field service would enhance the customer return [1]. After sales service are acknowledged as the source of firm's revenue, customer retention, and competitive advantage [2]. In certain manufacturing sector, after sales services and spare part may generate more than three times the turnover of the original purchase during the product life cycle [3]. In general, we can set spare part lifecycle in six different stages, with each stage having its individual characteristic [4].

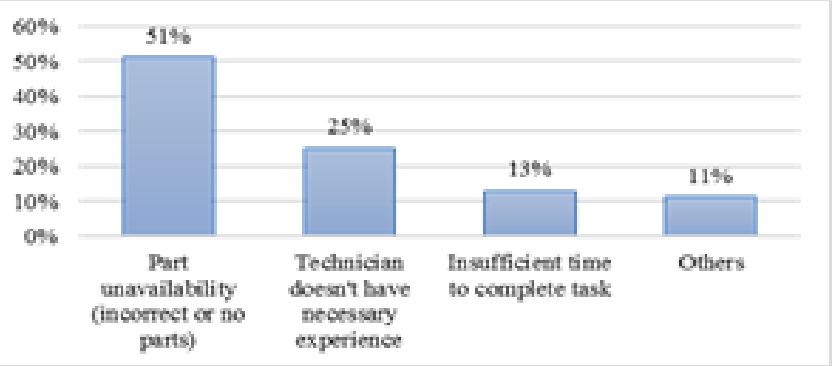

Source: Dutta (2013

Figure 1. Reasons for failure on first visit

TABLE I. RELATIONSHIP BETWEEN FIRST-TIME FIX PERFORMANCE AND CUSTOMER SATISFACTION

\begin{tabular}{llll}
\hline & $\begin{array}{l}\text { First-time } \\
<50 \%\end{array}$ & $\begin{array}{l}\text { First-time Fix } \\
<80 \%\end{array}$ & $\begin{array}{l}\text { First-time Fix } \\
>80 \%\end{array}$ \\
\hline $\begin{array}{l}\text { Customer } \\
\text { Satisfaction }\end{array}$ & $46 \%$ & $64 \%$ & $87 \%$ \\
\hline & & & Source: Dutta (2013
\end{tabular}

Spare part can be classified into the consumable and the repairable [5]. The repairable spare parts can be used many times through the recovery process, but the consumable spare parts are not repairable and usually scrapped when they have failed. Table 1 shows the relationship between the firsttime fixing performance and the customer satisfaction [1]. Clearly, it shows the importance of first-time fixing performance. Dutta [1] had pointed out that $51 \%$ of device repair failure was due to the lack of spare parts (see Fig. 1).

Fig. 2 illustrates the service network structure for the repairable spare part. It shows the importance of spare part utilization the complex global service operation. Defective 
parts are generally collected and shipped back by service point (called base) to the repair service center (called depot); after repaired, they are shipped back to service point for reuse.

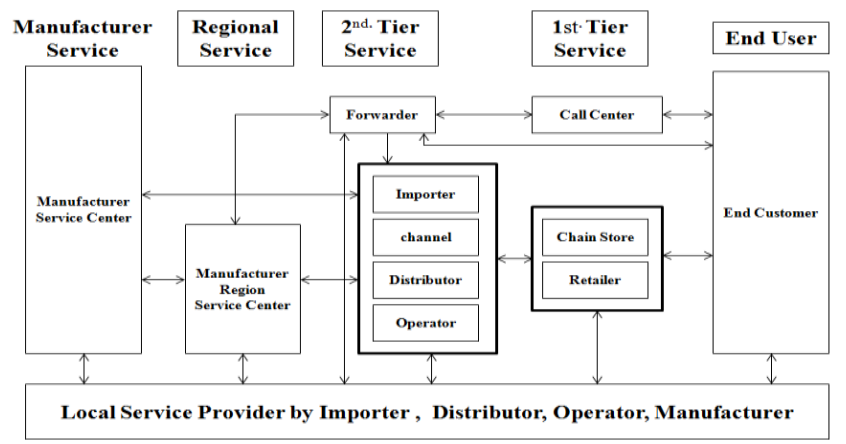

Figure 2. Service network structure

Most manufacturer repair service center would prefer the repaired defective spare parts be shipped back at a fix cycle time. Such fix cycle model simplifies the operations of the repair service center, though such arrangement will result in some spare part replenishment wastes due to higher buffer stocks. In order to understand the characteristics of the actual system, we examined the defect report of 11 mobile phone products for a period of 36 months as shown in Fig. 3. We found that the cause of such phenomenon may be due to the pipeline sales not evenly distributed, quality problem and the decline in demand during the out of warranty period. The defect up-down trend proves that a fix cycle arrangement will not be efficient because the repair service center workload is not evenly distributed.

In this study, a method using a flexible ship back time with least repair Turnaround Time (TAT) as the minimum replenishment cycle time is proposed. For convenience, we name the proposed method as LTAT method and the initial buffer as IB. When stock out time is forecasted, this method triggers the service point to ship the accumulated defective spare parts for repair. The objective is to arrange the repairreplenishment cycle so as to minimize the overall spare part inventory operation cost. For real industrial practice, this model also consider the last time buy (LTB) and the defect spare part condemnation rate (repair rate).

The study is organized in four sections, namely the introduction, the LTAT process, the simulation result, discussion and conclusion.

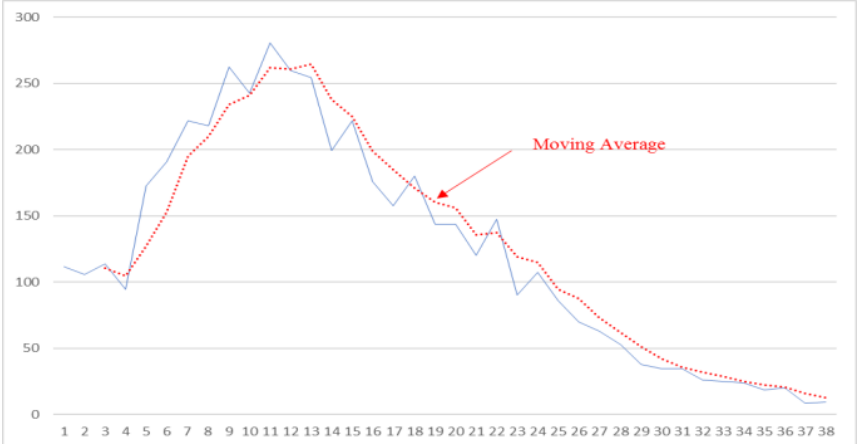

Figure 3. Trend for defective parts

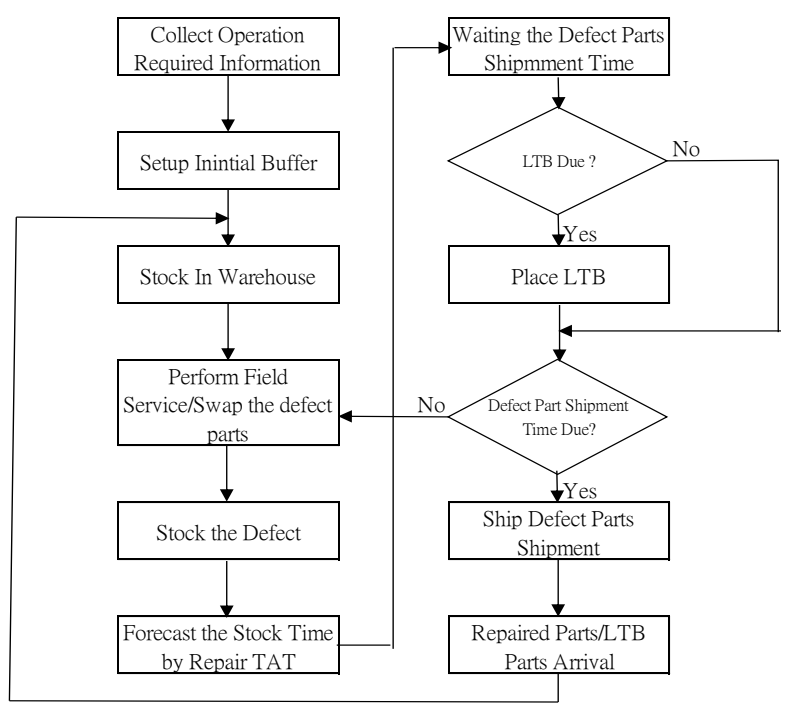

Figure 4. LTAT method

\section{LTAT PROCESS}

From [6], we develop a two-echelon repairable inventory model with infinite service center and service point repair capacity considering inventory for last time buy (LTB). The model consists of one service center and one service point operation. It assumes that service point stocks certain the weeks' inventory as initial buffer for saving ordering cost. It only allows one LTB to replenish the deficit of stock for the whole service period. The repair TAT is set to be the sum of shipping time between service point and service center, as well as the repair time in service center. The failure rate is assumed to be Poisson distribution. The goal of this study is to show that LTAT is a more efficient spare part model.

Forecasting the next stock out time is the key to LTAT method. Spare part failure rate is used to calculate the defective parts quantity in timeline. The remaining stock comes from the sum of last stock minus the current consumed quantity. The service center performs the repair and ships the repaired spare parts back to the service point. Certain percentage of the parts are lost (repair rate) in the repair process

The LTAT method is show is Fig. 4 and the notation is shown in Table 2. A simplified inventory model is formulated in equation (1). The objective of this method is to lower the related cost to improve spare part operation cost. A simplified inventory operation cost is formulated in equation (6).

In our simulation, the product is assumed to be sold within 6 months; it is shipped every month and each shipment has the same lot size. Warranty period is set to be one year. The out of warranty service rate is set to be $1 / 3$ of warranty failure rate. Spare part purchasing cost is US\$ 300 per parts, and order handling cost is US\$ 100 per order. Spare part carrying cost to be $25 \%$ of purchase cost per year based on [8]. Shipping cost with larger volume assume has $10 \%$ cost reduction (US\$ 0.5 and US\$ 0.45). Due to the 
purpose of this study is to prove LTAT method is feasible superior in performance, Microsoft EXCEL is enough to be the tool.

$$
C_{T}=C_{p}+C_{O}+C_{C}+C_{S}
$$

$$
C_{p}=C_{p u} \times Q_{p}
$$

$$
D F_{W K}=D I W_{W K}+D O W_{W K}
$$

$$
C_{o}=C_{p o} \times Q_{o}
$$

$$
D I W_{W K}=S I W_{W K} \times I W F R_{W K}
$$

$$
D O W_{W K}=S O W_{W K} \times O W F R_{W K}
$$

\begin{tabular}{|c|c|}
\hline Parameters & Description \\
\hline$w k$ & week $(w k=0,1 \ldots, W K)$ \\
\hline RTAT & Repair turnaround time, RTAT is set in 4 weeks. \\
\hline$W K$ & WK is ends at assigned period in Week \\
\hline$A O S_{w k}$ & $\begin{array}{l}\text { ASP (Authorized Service Party) on hand stock by } \\
\text { week }\end{array}$ \\
\hline$H R S_{w k}$ & Head Quarter Replenishment Shipment by week \\
\hline$H R S_{L T B}$ & $\begin{array}{l}\text { Head Quarter Replenishment Shipment-last time } \\
\text { buy }\end{array}$ \\
\hline$D F_{w k}$ & Defect parts by week \\
\hline$D I W_{w k}$ & In warranty defect parts by week \\
\hline$D O W_{w k}$ & Out of warranty defect parts by week \\
\hline$S I W_{w k}$ & Sales quantity still in warranty \\
\hline$S O W_{w k}$ & Sales quantity is out of warranty \\
\hline$I W F R_{w k}$ & $\begin{array}{l}\text { In warranty failure rate by week (Annual failure } \\
\text { rate/52) }\end{array}$ \\
\hline$O W F R_{w k}$ & $\begin{array}{l}\text { Out of warranty failure rate by week (Annual } \\
\text { failure rate/52) }\end{array}$ \\
\hline$C_{T}$ & Cost for total spare part operation \\
\hline$C_{p}$ & Cost for spare part purchasing \\
\hline$C_{o}$ & Cost for order process \\
\hline$C_{c}$ & Cost for carry cost \\
\hline$C s$ & Cost for shipping \\
\hline$C_{p u}$ & Cost per spare part \\
\hline$C_{p o}$ & Cost per order issue \\
\hline$C_{c y}$ & Cost for spare part carrying one year $\left(25 \%\right.$ of $\left.\mathrm{C}_{\mathrm{pu}}\right)$ \\
\hline$C_{p s}$ & Cost per shipping spare part \\
\hline$\underline{Q_{p}}$ & Quantity of spare part purchase \\
\hline$Q_{o}$ & Quantity of order issue \\
\hline$Q_{s}$ & Quantity of spare part ship \\
\hline$T_{h}$ & Spare part holding period \\
\hline
\end{tabular}

$$
C_{c}=C_{p y} \times T_{h}
$$

$$
H R S_{W K}=D F_{W K-R T A T} \times(1-H R S)
$$

TABLE II. NOTATIONS
$C_{s}=C_{p s} \times Q_{s}$ 
TABLE III. THE FORECASTING AND REPLENISHMENT

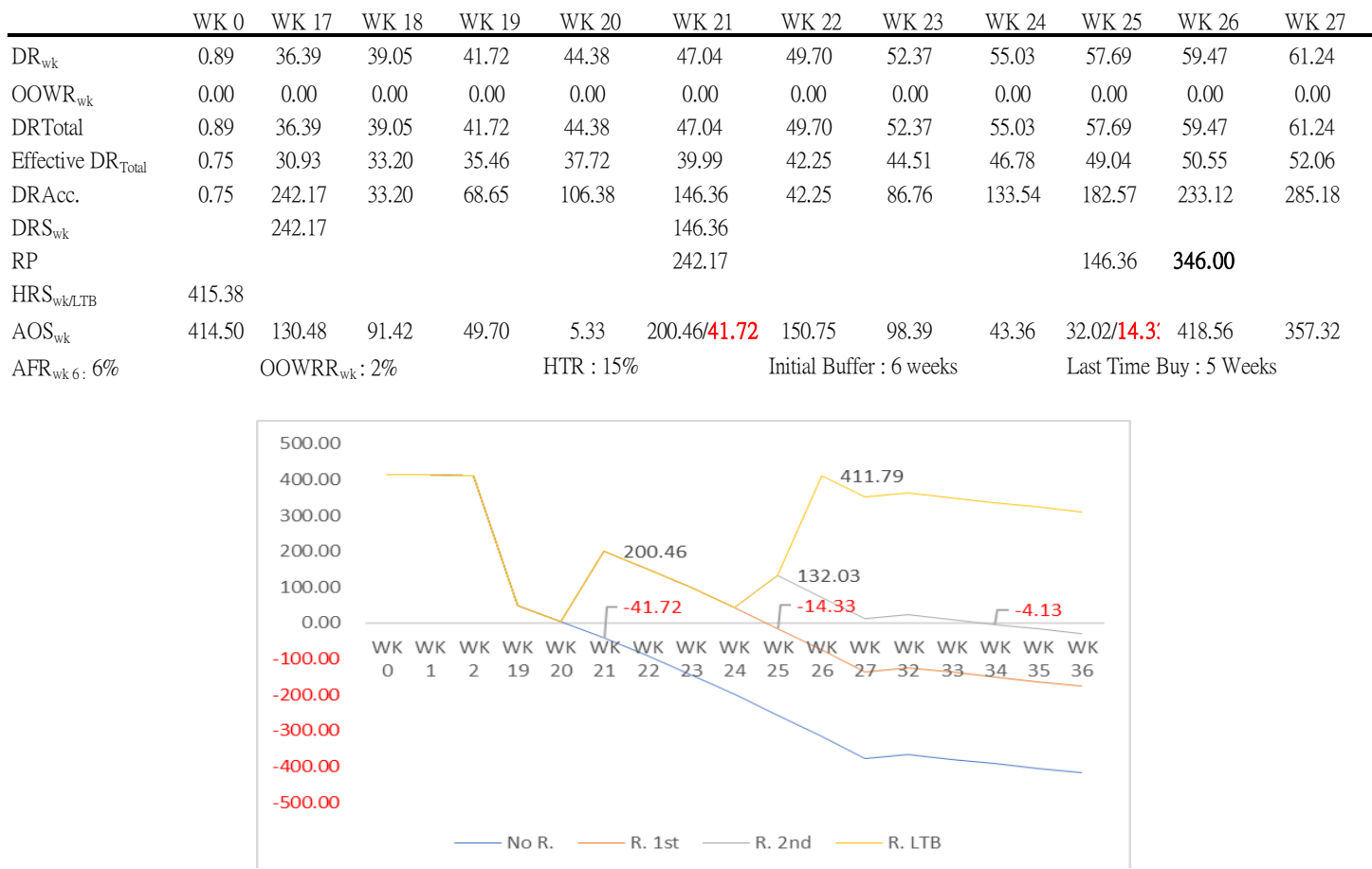

Figure 5. Spare part inventory transits

From Table 3 and Fig. 5, the forecasting result, the first stock out time occurs at week 21 and the shortage quantity is $41.72\left(\mathrm{AOS}_{21}\right)$. The service point must send the defective parts under the minimum repair TAT (4 weeks) at week 17 for repair so that parts will be repaired and shipped back on week 21 . When the repaired parts arrive, the $\mathrm{AOS}_{21}$ changes to a quantity of 200.46. Similar situation happens at week 25 (14.33 and 132.03) and at LTB arrives week 26, and this will be repeated until the service period is completed.

\section{THE SimULATION RESUlT}

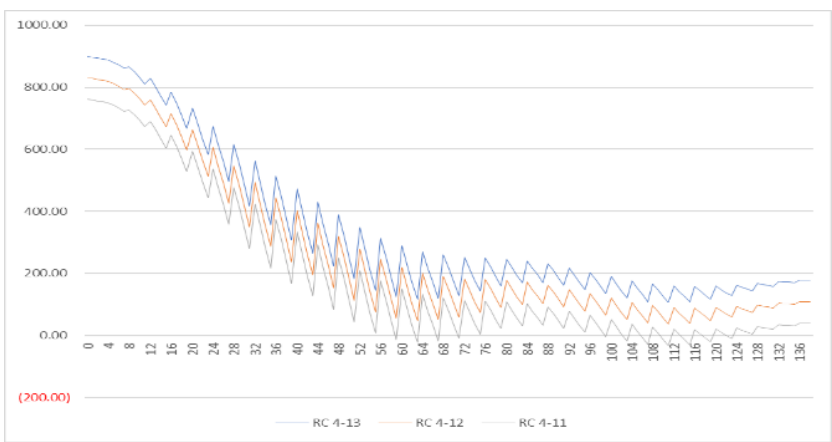

Figure 6. Fix defect parts return cycle

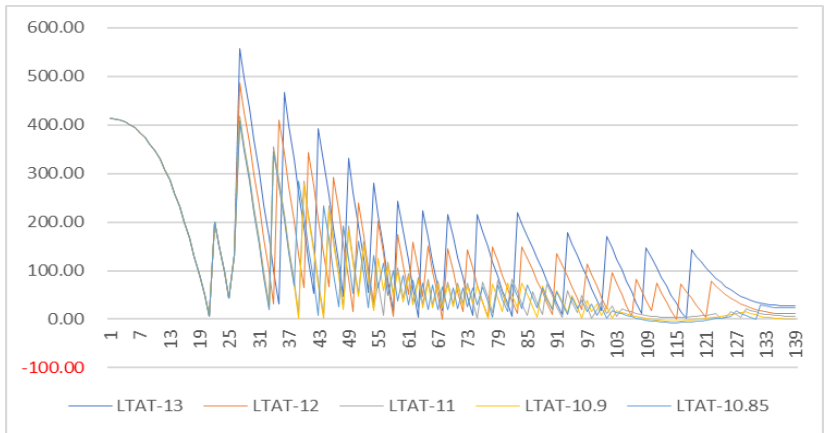

Figure 7. Flexible defect parts return cycle

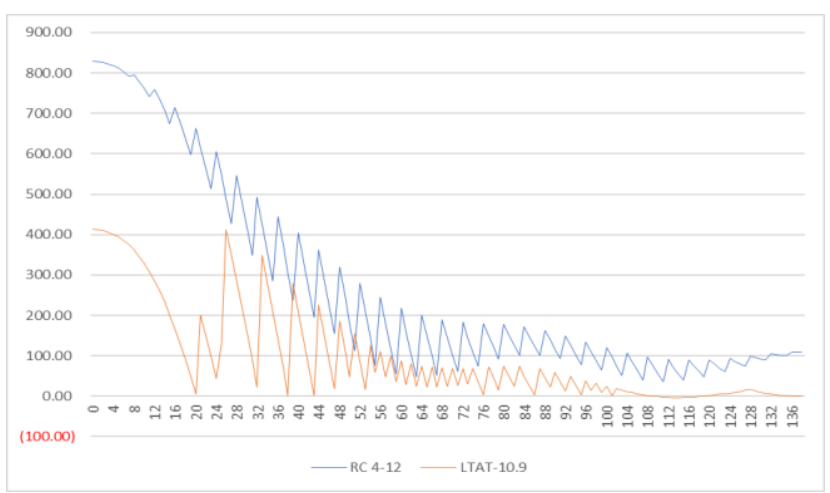

Figure 8. Fix vs. flexible no stock out

This section compares the performance of fix cycle model to LTAT based on the simulation result. First, Fig. 6 
shows when fix cycle model has initial buffer level above 12 weeks, there is no stock out occurs. In the meantime, LTAT performance as shown in Fig. 7 tells when the initial buffer level is set at 6 weeks with LTB 4.95 weeks' quantity; there is no stock out as well. This result clear indicates that LTAT model can provide no stock out under lower initial buffer. Furthermore, by observing these two models' behavior shown in Fig. 8, we found that LTAT model needs less replenishment cycle compare to fix model (40 vs 31 ). Table 4 summarizes the operation cost calculation of these two models based on equation (6). The result shows that LTAT model's operation cost is US\$ 45,178.35 (11.97\%) lower than the fix cycle model.

Focusing on the replenishment cycle times, we compare fix model with 12 weeks' initial buffer and LTAT model with 6 weeks' initial buffer together with 6 weeks LTB as shown in Table 5. Table 5 still indicates flexible model has advantages both in replenishment cycle (23 vs. 31) and operation cost (US\$ $16,330.77$ or $4.33 \%$ ). By these facts, it concludes that flexible model even when runs higher replenishment times, the benefit in lower initial buffer still drives operation cost is firmed.

TABLE IV.

FLEXIBLE MODEL BENEFIT (IB: 10.95)

\begin{tabular}{|c|c|c|c|c|c|c|}
\hline \multirow{3}{*}{ Costs/IB week } & \multirow{3}{*}{ Cost In USD } & \multicolumn{5}{|c|}{12 vs. 10.95} \\
\hline & & \multicolumn{2}{|r|}{ Fix Period } & \multicolumn{2}{|c|}{ Flexible Period } & \multirow{2}{*}{ Benefit } \\
\hline & & Qty. & Sub-Total & Qty. & Sub-Total & \\
\hline Purchase Cost/piece & US $\$ 300.00$ & \begin{tabular}{|l|}
831 \\
\end{tabular} & \begin{tabular}{|l|} 
US $\$ 249,230.77$ \\
\end{tabular} & \begin{tabular}{|l|l|}
757 \\
\end{tabular} & US $\$ 227,100.00$ & \multirow{5}{*}{ US $\$ 45,178.35$} \\
\hline Carrying Cost/year & US\$75.00 & 831 & \begin{tabular}{|l} 
US $\$ 124,650.00$ \\
\end{tabular} & 757 & US $\$ 100,739.42$ & \\
\hline Order Cost/order & US $\$ 100.00$ & 31 & US $\$ 3,100.00$ & 40 & US $\$ 4,000.00$ & \\
\hline Shipping Cost/piece & US\$0.50 & 831 & US $\$ 415.50$ & 757 & US $\$ 378.50$ & \\
\hline Sub-Total & & & \begin{tabular}{|l|} 
US $\$ 377,396.27$ \\
\end{tabular} & & US $\$ 332,217.92$ & \\
\hline
\end{tabular}

TABLEV. FiX AND FLEXIBLE MODEL WiTH 12 WeEks INITIAL BufFeR

\begin{tabular}{|c|c|c|c|c|c|c|}
\hline \multirow{3}{*}{ Costs/IB week } & \multirow{3}{*}{\begin{tabular}{|c|} 
Cost In USD \\
Fix Period \\
\end{tabular}} & \multicolumn{5}{|c|}{12 vs. 12} \\
\hline & & \multicolumn{2}{|r|}{ Fix Period } & \multicolumn{2}{|c|}{ Flexible Period } & \multirow{2}{*}{ Benefit } \\
\hline & & Qty. & Sub-Total & Qty. & Sub-Total & \\
\hline Purchase Cost/piece & US $\$ 300.00$ & 831 & US $\$ 249,230.77$ & 831 & US\$249,300.00 & \multirow{5}{*}{ US $\$ 16,330.77$} \\
\hline \begin{tabular}{|l|} 
Carrying Cost/year \\
\end{tabular} & US\$75.00 & 831 & US $\$ 124,650.00$ & 831 & US $\$ 109,050.00$ & \\
\hline Order Cost/order & US\$100.00 & 31 & US $\$ 3,100.00$ & 23 & US $\$ 2,300.00$ & \\
\hline Shipping Cost/piece & US\$0.50 & 831 & US $\$ 415.50$ & 831 & US $\$ 415.50$ & \\
\hline \begin{tabular}{|l|} 
Sub-Total \\
\end{tabular} & & & US $\$ 377,396.27$ & & US $\$ 361,065.50$ & \\
\hline
\end{tabular}

\section{CONCLUSION}

This study shows that LTAT model is more effective in managing spare part inventory operation by lowering the operation cost and the replenishment cycle. We also consider last time buy and spare part repair rate in the simulation. Another benefit of this method is its simplicity in understanding and operation. For future research, we can consider initial buffer and last time buy quantity.

\section{REFERENCES}

[1] S. Dutta, "Fixing First-Time Fix: Repair Field Service Efficiency to Enhance Customer Return", Aberdeen Group, 2013.

[2] N. Saccani, P. Johansson, and M. Perona, "Configuring the after-sales service supply chain: A multiple case study", International Journal of Production Economics, vol. 110, 2007, pp. 52-69.

[3] R. Wise and P. Baumgartner, "Go downstream-The new profit imperative in manufacturing", Harvard Business Review, vol. 77 (5), 1999, pp. 133-141.

[4] R. Solomon, P. Sandborn, and M. Pecht, "Electronic part life cycle concpets and obsolescence forecasting", IEEE Trans. On Components and Packaging Technologies, Dec. 2000, pp. 707-717.

[5] M. A.Cohen, Y. Zheng, and V. Agrawal, "Service parts logistics: a benchmark analysis", IIE Transactions, vol. 29 (8), 1997, pp. 627-639.

[6] D. R. Guide and R. Srivastava, "Repairable inventory theory: Models and applications", European Journal of Operational Research, vol. 102 (1), 1997, pp.1-20.

[7] S. C. Graves, "A multi-echelon inventory model for a repairable item with one-for-one replacement", Management Science, vol. 31 (10), 1985, pp. 1247-1256.

[8] M.E. Trimp, R. Dekker, and R. H. Teunter, "Optimise initial spare parts inventories: an analysis and improvement of an electronic decision tool", Report Econometric Institute EI 2004-52, Erasmus University, 2004. 